\title{
QUALIDADE PÓS-COLHEITA DO MAMÃO FORMOSA ARMAZENADO SOB REFRIGERAÇÃO ${ }^{1}$
}

\author{
RAILENE HÉRICA CARLOS ROCHA², SELMA ROGÉRIA DE CARVALHO NASCIMENTO³, JOSIVAN BARBOSA \\ MENEZES ${ }^{4}$, GLAUBER HENRIQUE DE SOUZA NUNES ${ }^{5}$, EBENÉZER DE OLIVEIRA SILVA ${ }^{6}$
}

RESUMO - O objetivo deste trabalho foi avaliar a qualidade e vida útil pós-colheita do mamão Formosa 'Tainung 01' armazenado sob temperaturas refrigeradas. Os frutos foram provenientes de um plantio comercial no município de Baraúna-RN. O armazenamento ocorreu em câmaras a 8; 10 e $12^{\circ} \mathrm{C}$ $\pm 1{ }^{\circ} \mathrm{C}$, mantidas a $90 \pm 5 \%$ UR durante os períodos de 7; 14; 21 e 28 dias. Após cada período, os mesmos foram transferidos para a condição ambiente ( $20 \pm 1^{\circ} \mathrm{C} 60 \pm 5 \%$ UR) onde se simulou um período de prateleira de sete dias. Em seguida, os mamões foram avaliados quanto à aparência externa e interna, coloração da casca, perda de massa e firmeza de polpa. A melhor estimativa para a manutenção da qualidade e aumento da vida útil póscolheita do mamão Formasa 'Tainung 01' foi observada nos frutos submetidos a 10 ${ }^{\circ} \mathrm{C}, 90 \pm 5 \%$ UR até 20 dias, com boa aparência externa e interna (notas acima de 3,0), desenvolvimento da coloração amarela na casca, firmeza de polpa superior a $20 \mathrm{~N}$ e perda de massa de 7\%. Os frutos submetidos a $8^{\circ} \mathrm{C}$ e a $10^{\circ} \mathrm{C}, 90 \pm 5 \%$ UR desenvolveram sintomas de danos pelo frio após 21 dias de armazenamento, agravando-se com a extensão do armazenamento. No armazenamento a $12^{\circ} \mathrm{C}, 90 \pm 5 \% \mathrm{UR}$, observou-se um amadurecimento acelerado com amolecimento aquoso da polpa a partir de 28 dias. Aos 35 dias, os frutos estavam inaptos para o consumo, havendo grande incidência de Colletotrichum gloeosporioides.

Termos para indexação: Carica papaya L., temperatura controlada, conservação.

\section{POST HARVEST QUALITY OF PAPAYA STORAGE UNDER REFRIGERATION}

\begin{abstract}
The purpose of this study was to evaluate the post harvest quality and shelf storage of 'Tainung 01' papaya fruit stored under different refrigerated temperatures. The fruits were from a commercial cultivation in the district of Baraúna-RN-Brazil. The fruits were storied in cold rooms at $8^{\circ}, 10^{\circ}$ and $12^{\circ} \mathrm{C} \pm 1{ }^{\circ} \mathrm{C}$ and $90 \pm 5 \% \mathrm{RH}$ during the period of $7,14,21$ e 28 days. After each period, the fruits were put at environmental conditions $\left(20^{\circ} \pm 1^{\circ} \mathrm{C} 60 \pm 5 \% \mathrm{RH}\right)$ where was simulated a shelf life period for seven days. Then, the papayas were analyzed in relationship to the external and internal appearance, coloration of the skin, mass loss and pulp firmness. The best evaluation for quality maintenance and increase the post harvest usefue life of the ' Tainung 01' papaya was observed in the fruits submitted to $10^{\circ} \mathrm{C}, 90 \pm 5 \%$ RH up to 20 days, with good extern and interns appearance (notes above 3,0), development of the yellow coloration in the skin, firmness of superior pulp of $20 \mathrm{~N}$ and loss of mass of $7 \%$. The submitted fruits at $8^{\circ} \mathrm{C}$ and to $10^{\circ} \mathrm{C}, 90 \pm 5 \%$ RH developed symptoms of chilling injury after 21 days of storage, becoming worse with the extension of the storage. In the storage to $12^{\circ} \mathrm{C}, 90 \pm 5 \% \mathrm{RH}$ an accelerated ripening was observed with aqueous softening of the pulp starting from 28 days. On the $35^{\text {th }}$ day, the fruits were inapt for consumption having great incidence of Colletotrichum gloeosporioides.
\end{abstract}

Index terms: Carica papaya L., controled temperature, conservation.

\section{INTRODUÇÃO}

Originado na América, o mamoeiro (Carica papaya L.) é cultivado em mais de 40 países, sendo o Brasil o principal produtor mundial (FAO, 2004). A produção nacional de mamão baseia-se nos grupos Formosa e Havaí, sendo este último comercializado tanto no mercado interno quanto no externo, enquanto o Formosa destina-se principalmente ao mercado interno.

Para o consumo in natura do mamão Formosa, são necessárias operações adicionais, como corte e acondicionamento. O fruto minimamente processado torna o consumo muito prático, e além de agregar valor ao produto, este poderá ser consumido com facilidade nos mais diferentes ambientes e permitir um melhor aproveitamento final (TEIXEIRA et al., 2001).

Todos os procedimentos pós-colheita do Formosa são realizados sob temperatura ambiente. Na comercialização a granel, a qualidade é comprometida por danos mecânicos, como arranhões, cortes e abrasões, que favorecem a incidência de doenças aumentando, conseqüentemente, as perdas. Sob temperatura ambiente $\left(27,4{ }^{\circ} \mathrm{C}\right)$ a vida útil pós-colheita é estimada em seis dias, ocorrendo, posteriormente, murchamento e intensa infestação de patógenos (Ribeiro, 2002).

Em mamão Formosa, até o presente momento, não se tem conhecimento da temperatura que possibilite a manutenção dos atributos de qualidade, como aparência, textura, valor nutricional, aroma e sabor, e retarde a incidência de podridões causadas por Botryodiplodia sp, Colletotrichum sp., Fusarium sp., Phoma sp. e Rhizopus sp.

O objetivo deste trabalho foi avaliar a qualidade e a vida útil pós-colheita do mamão Formosa 'Tainung 01', armazenado sob refrigeração.

\section{MATERIAL E MÉTODOS}

Os frutos do mamoeiro Formosa 'Tainung 01' foram obtidos em pomar comercial no município de Baraúna - RN, localizado no Agropólo Mossoró-Assu. A colheita foi realizada pela manhã, no estádio de maturação 'color break', com 10\% de coloração amarela na casca. Posteriormente, foram acondicionados em caixas plásticas e transportados à temperatura de $20^{\circ} \mathrm{C}$ para o Laboratório de Pós-Colheita da Escola Superior de Agricultura de Mossoró (ESAM), onde uma amostragem de dez frutos foi caracterizada por ocasião da colheita (Tabela 1), sendo os demais frutos previamente tratados, por imersão, em água contendo o fungicida procloraz, na concentração de $300 \mathrm{mg} \cdot \mathrm{L}^{-1}$, durante 3 minutos. Em seguida, o experimento foi instalado em delineamento inteiramente casualizado, em esquema fatorial $3 \times 4$ (temperatura $\mathrm{x}$ tempo de armazenamento), com cinco repetições e dois frutos por parcela. $\mathrm{O}$ armazenamento foi realizado em câmaras refrigeradas a $8 ; 10$ e $12^{\circ} \mathrm{C} \pm 1$ ${ }^{\circ} \mathrm{C}$ e $90 \pm$ 5\% UR durante 7; 14; 21 e 28 dias. Após cada período, foi simulado o período de comercialização durante sete dias sob $20 \pm 1^{\circ} \mathrm{C}$ e $60 \pm 5 \%$ UR. Foram avaliadas as seguintes características de qualidade: Aparências externa e interna - com a utilização de escalas

\footnotetext{
${ }^{1}$ (Trabalho 073/2005). Recebido: 20/02/2005. Aceito para publicação: 06/10/2005.

2 Parte da dissertação de mestrado do primeiro autor apresentada ao Curso de Fitotecnia da ESAM, c. p. 137, CEP 59.625-900, Mossoró-RN, e-mail: raileneherica@hotmail.com.

${ }^{3}$ Eng. Agr ${ }^{0}$. Prof. D.Sc., ESAM - Dept ${ }^{0}$ Fitossanidade, c. p. 137, CEP 59.625-900, Mossoró-RN, e-mail: selma@esam.br.

${ }^{4}$ Eng. Agr ${ }^{\circ}$. Prof. D.Sc., ESAM - Dept ${ }^{\circ}$ Química e Tecnologia, c. p. 137, CEP 59.625-900, Mossoró-RN, e-mail: josivan@esam.br.

${ }^{5}$ Eng. Agr ${ }^{\circ}$. Prof. D.Sc., ESAM - Dept ${ }^{0}$ Fitotecnia, c. p. 137, CEP 59.625-900, Mossoró-RN.

${ }^{6}$ Eng. Agr ${ }^{\circ}$. DSc.Pesquisador - EMBRAPA - Agroindústria Tropical, Fortaleza-CE, e-mail: ebenezer@cnpat.embrapa.br.
} 
TABELA 1 - Caracterização inicial do mamão Formosa ‘Tainung 01’ por ocasião da colheita.

Cor da Casca (notas de 1 a 5)

Aparência externa (notas de 1 a 5)

Aparência interna (notas de 1 a 5)

Comprimento Longitudinal $(\mathrm{cm})$

Comprimento Transversal $(\mathrm{cm})$

Espessura de Polpa $(\mathrm{cm})$

Peso $(\mathrm{g})$

\begin{tabular}{l}
1,0 \\
5,0 \\
5,0 \\
22,71 \\
9,84 \\
3,06 \\
181,2 \\
\hline
\end{tabular}

subjetivas de notas adaptadas de Menezes (1998), sendo:1=fruto extremamente deteriorado; $2=$ severa deterioração; $3=$ média deterioração; 4= leve deterioração; 5= ausência de deterioração. Frutos com notas iguais ou inferiores a 3 foram considerados impróprios para consumo. Observou-se, na aparência externa: danos pelo frio, depressões, manchas, murcha e manifestações de doenças, e na aparência interna: amolecimento aquoso da polpa, endurecimento interno provocado por dano pelo frio e manifestação de doenças;

Coloração da casca - estabelecida de acordo com a escala de notas subjetiva proposta por Wills \& Widjanarko (1995);

Perda de massa - expressa em porcentagem, considerando-se a diferença entre a massa inicial do fruto e aquela obtida ao final de cada período de armazenamento;

Firmeza da polpa - efetuada em regiões equatoriais da superfície do fruto inteiro, desprovido de casca, utilizando-se de um penetrômetro Mc Cormick modelo FT 327, com ponteira de 7,8 mm de diâmetro, sendo os resultados expressos em Newton $(\mathrm{N})$;

Presença de patógenos - Quando os frutos apresentaram crescimento fúngico, o isolamento foi realizado diretamente através da transferência do crescimento dos patógenos, com o auxílio de uma alça reta flambada, para as placas de Petri contendo meio de crescimento BDA (batata-dextrose-ágar), acrescido de $0,01 \mathrm{~g}$ tetraciclina por litro. Quando do não-aparecimento, realizou-se isolamento da porção do fruto infectado, retirando-se fragmentos superficiais das margens das lesões. Estes foram submetidos em álcool etílico 70\%, durante 30 segundos, e em hipoclorito de sódio $2 \%$, durante 60 segundos, lavados em água destilada, enxugados em papel de filtro esterilizado e transferidos para as placas de Petri contendo o meio de crescimento. As placas foram mantidas em câmara tipo B.O.D. à temperatura de $26^{\circ} \mathrm{C} \pm 1^{\circ} \mathrm{C}$ para proporcionar o crescimento dos fungos e posterior identificação.

As análises de variância das características avaliadas foram realizadas através do software SAS (SAS Institute Inc., 1993). As regressões foram realizadas com o programa Table Curve (Jandel Scientific, 1991).

\section{RESULTADOS E DISCUSSÃO}

Constatou-se perda de qualidade relacionada à aparência externa dos frutos com o aumento do período de armazenamento e das temperaturas. As melhores aparências externas foram observadas em frutos submetidos à temperatura de $8 \mathrm{a} 10^{\circ} \mathrm{C}$, com notas superiores a 3,0, até 20 dias de armazenamento (Figura 1).

No geral, a qualidade do mamão Formosa foi reduzida devido a danos pelo frio, manchas de senescenência, murchamento e presença da mancha fisiológica do mamoeiro, também conhecida como 'sarda', nas temperaturas de $10 \mathrm{e} 12^{\circ} \mathrm{C}$. Através do isolamento dos patógenos, verificouse a presença de Cladosporium sp., Penicillium sp. e Fusarium sp. Aos 35 dias, verificou-se, nos frutos submetidos a $12^{\circ} \mathrm{C}$, o sintoma mais severo de doença causado pelo fungo Colletotrichum gloeosporioides, que ocasionou manchas marrom-claras, circulares e deprimidas, que se aprofundavam na polpa, tornando o fruto inadequado para o consumo.

Observou-se dano pelo frio em alguns frutos submetidos a $8 \mathrm{e}$ $10^{\circ} \mathrm{C}$ a partir do $21^{\circ} \mathrm{dia}$ de armazenamento, caracterizado por escaldadura e diminuição do avanço da cor amarela na casca, que se agravou com o aumento do período de armazenamento. Resultados semelhantes foram observados por Silva (1995), em mamão ‘Sunrise Solo’ Improved Line
72/12, armazenado a $8^{\circ} \mathrm{C}$ por 21 dias, acrescido por cinco dias a $22,5^{\circ} \mathrm{C}$. Houve perda de qualidade dos frutos, relacionada à aparência

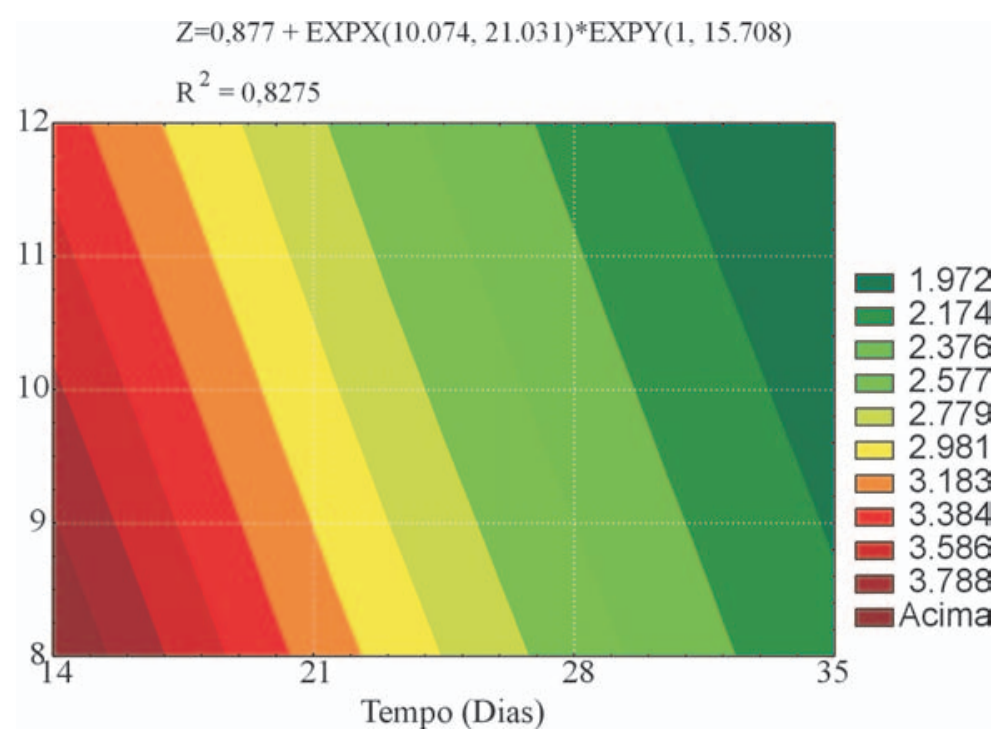

FIGURA 1 - Aparência externa (notas de 1 a 5) em mamão Formosa 'Tainung 01' mantido a 8; 10 e $12^{\circ} \mathrm{C}$ e $90 \pm 5 \%$ UR durante os períodos de 7; 14; 21 e 28 dias, acrescidos de um período de comercialização simulado de 7 dias a $20^{\circ} \mathrm{C}$ e 65 $\pm 5 \%$ UR.

interna com o aumento do período de armazenamento. Observou-se, nas temperaturas entre 10 e $12^{\circ} \mathrm{C}$, maior manutenção de qualidade, com notas superiores a 3,0 até $\mathrm{o} 32^{\circ}$ dias de armazenamento. $\mathrm{A} 8^{\circ} \mathrm{C}$, a qualidade interna para consumo foi de 23 dias (Figura 2), constatando-se dano pelo frio, caracterizado pela inibição do amadurecimento, polpa endurecida ao toque e sabor amargo.

As mudanças mais significativas para a coloração da casca foram verificadas entre as temperaturas de 8 a $10^{\circ} \mathrm{C}$ a partir do $21^{\circ}$ dia. $A 8^{\circ} \mathrm{C}$, verificaram-se diminuição do avanço da cor amarela da casca e inibição do amadurecimento dos frutos com o aumento do tempo de armazenamento. Em temperaturas superiores a $10^{\circ} \mathrm{C}$, houve desenvolvimento da coloração amarela da casca, em todos os tempos de armazenamento (Figura 3). Sob condições ambientais, Ribeiro (2002) verificou evolução na coloração da casca em mamão Formosa 'Tainung 01'até o $6^{\circ}$ dia de armazenamento, estando os frutos ótimos para a comercialização entre o $3^{\circ}$ e o $6^{\circ}$ dia, quando apresentavam mais de $50 \%$

$$
\begin{aligned}
& Z=5,967+(-1,182) x+0,041 x^{2}+(-0,001) x^{3}+1,65 y+(-0,075) y^{2} \\
& R^{2}=0,9496
\end{aligned}
$$

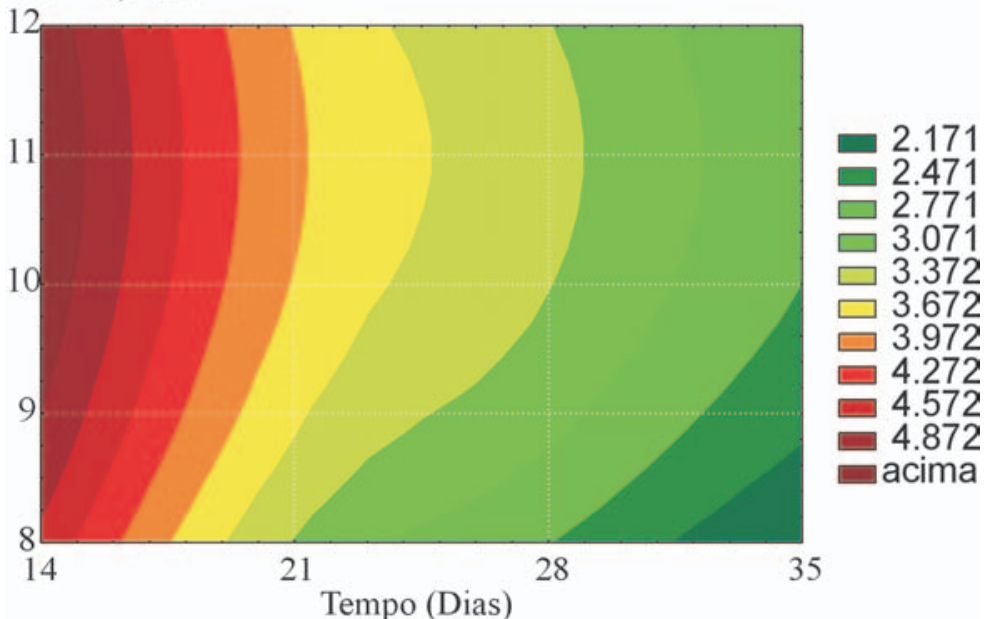

FIGURA 2 - Aparência interna (notas de 1 a 5) em mamão Formosa 'Tainung 01' mantido a $8 ; 10$ e $12^{\circ} \mathrm{C}$ e $90 \pm 5 \%$ UR durante os períodos de 7; 14; 21 e 28 dias, acrescidos de um período de comercialização simulado de 7 dias a $20^{\circ} \mathrm{C}$ e 65 $\pm 5 \%$ UR. 
da cor da casca amarela. Em mamão ‘Sunrie Solo’ armazenado à temperatura ambiente, Lopes (1997) verificou evolução na coloração da casca dos frutos até o $8^{\circ}$ dia. Sob condições refrigeradas, Abeles et al., (1992) e Wills et al., (1981) observaram que, ao reduzir a temperatura, a degradação de clorofila diminuiu, como conseqüência da redução da produção de etileno, da ação combinada de clorofilases e sistemas oxidativos e da redução do pH pela liberação de ácidos orgânicos do vacúolo celular, o que pode ter proporcionado o aspecto verdoso nos frutos a $8^{\circ} \mathrm{C}$.

Houve incremento na perda de massa com o aumento do período de armazenamento e das temperaturas. As menores perdas de massa foram encontradas entre as temperaturas de 8 a $10^{\circ} \mathrm{C}$, até o $23^{\circ}$ dia. No $14^{\circ}$ dia, foram observadas, nas temperaturas superiores a $10^{\circ} \mathrm{C}$, as maiores perdas de massa, acima de 5,7\% (Figura 4).

$\mathrm{Na}$ temperatura de $12^{\circ} \mathrm{C}$, aos 28 dias de armazenamento, constatou-se murchamento dos frutos na região próxima ao pedúnculo, havendo perda de massa de aproximadamente 10\% (Figura 4). Aos 35 dias, foi verificado murchamento nos frutos mantidos sob todas as temperaturas.

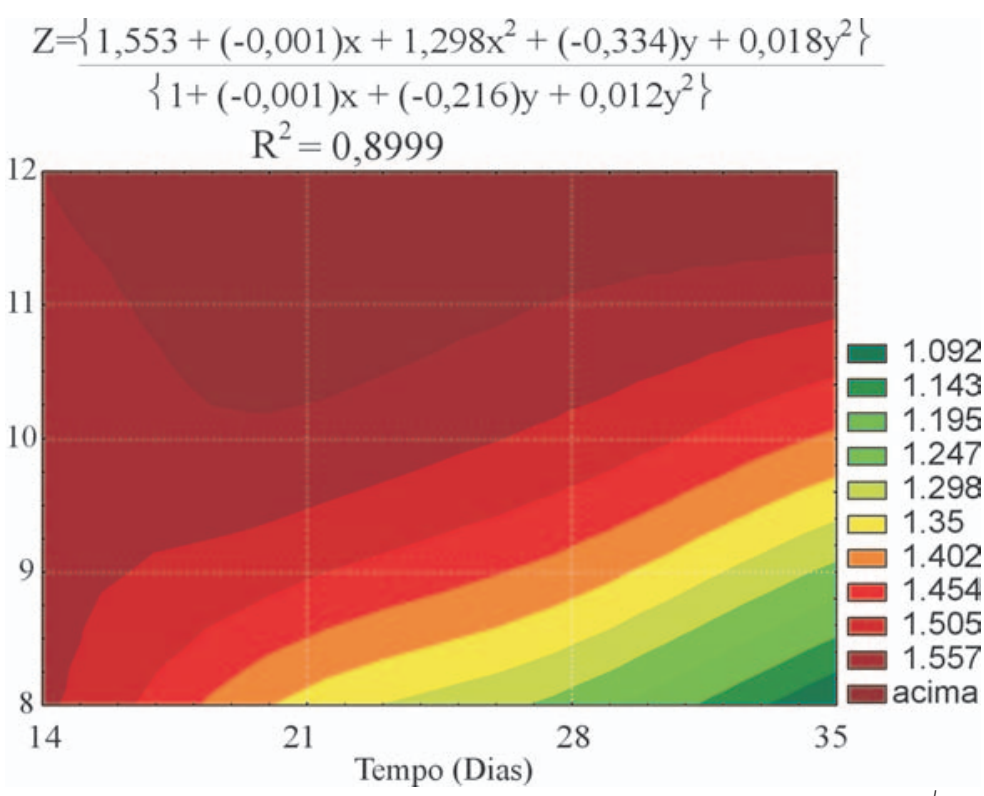

FIGURA 3 - Cor da casca (notas de 1 a 5, dados transformados em $\sqrt{\mathrm{x}}+$ 0,5) em mamão Formosa 'Tainung 01' mantido a 8; 10 e $12^{\circ} \mathrm{C}$, e $90 \pm 5 \%$ UR durante os períodos de 7; 14; 21 e 28 dias, acrescidos de um período de comercialização simulado de 7 dias a $20^{\circ} \mathrm{C}$ e $65 \pm 5 \%$ UR.

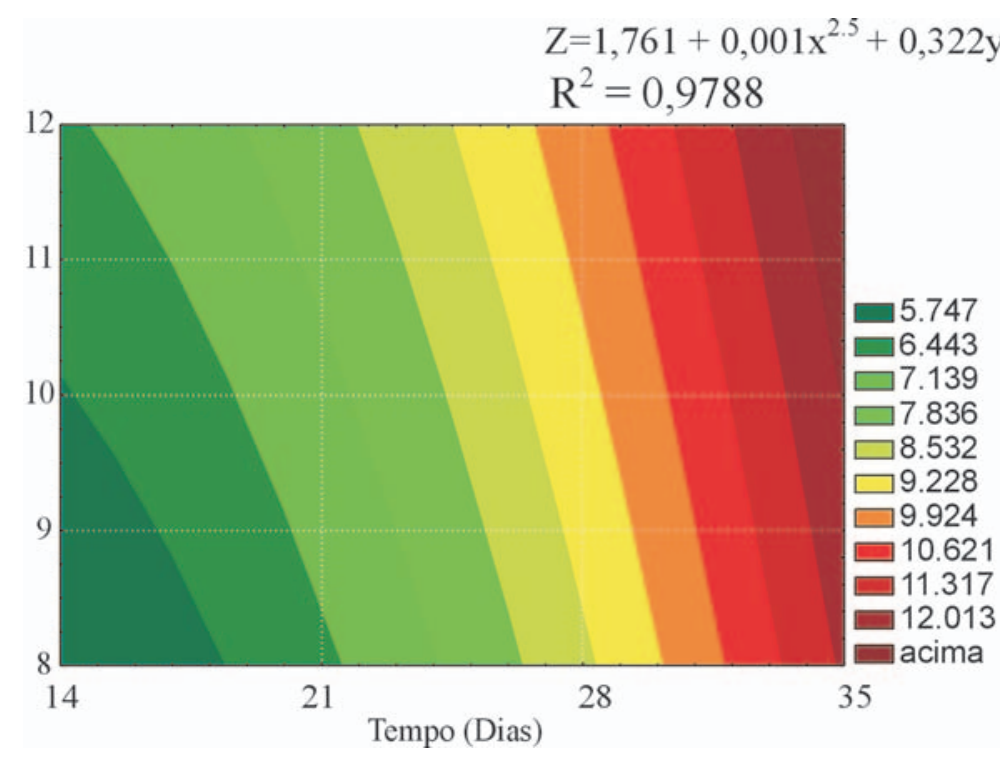

FIGURA 4 - Perda de massa (\%) em mamão Formosa 'Tainung 01' mantido a $8 ; 10$ e $12^{\circ} \mathrm{C}$ e $90 \pm 5 \%$ UR durante os períodos de 7; 14; 21 e 28 dias, acrescidos de um período de comercialização simulado de 7 dias a $20^{\circ} \mathrm{C}$ e $65 \pm 5 \%$ UR.
Em estudos desenvolvidos com mamão 'Sunrise Solo', verificou-se perda de massa média de 14,19\% sob condições ambientais até o $8^{\circ}$ dia de armazenamento, quando estavam impróprios para a comercialização por apresentarem distúrbios caracterizados por enrugamento acentuado, depressões e manchas escuras na casca (Lopes, 1997). Neste trabalho, a maior perda de massa foi acima de $12 \%$ ao final de 35 dias de armazenamento, a $12^{\circ} \mathrm{C}$ (Figura 4), sendo também inferior à obtida por An \& Paul (1990), com mamão papaya, que foi de $13 \%$ ao final de dez dias de armazenamento a $30^{\circ} \mathrm{C}$. Este fato justifica o uso da refrigeração associada à umidade relativa do ar, o que proporciona a redução da atividade respiratória e da energia liberada para o meio, na forma de calor, a redução do déficit de pressão de vapor e, conseqüentemente, a perda de água por transpiração, o que implica a perda de massa e a qualidade dos frutos.

Houve aumento na firmeza, provocado pelo endurecimento de polpa, com a redução da temperatura em todo o período de armazenamento. Os maiores valores foram observados nos frutos acondicionados a $8^{\circ} \mathrm{C}$, no período de 18 a 35 dias (Figura 5). Este aumento é explicado pelo retardo no amadurecimento devido a danos pelo frio, que promoveu o endurecimento interno da polpa, observado aos 21 dias de armazenamento. Fato semelhante foi constatado por Silva (1995) em mamão refrigerado, onde frutos com maior severidade de danos pelo frio tiveram seu amadurecimento totalmente inibido. De acordo com Harvery \& Chan (1988), há uma correlação significativa entre a sensibilidade a danos pelo frio, provocada por escaldadura na casca, e o endurecimento interno da polpa. Segundo os autores, tais sintomas diminuem com a redução do tempo de exposição à baixa temperatura e com o avanço da maturação dos frutos. Na faixa de temperatura entre 10 e $12^{\circ} \mathrm{C}$, observaram-se os menores valores para a firmeza.

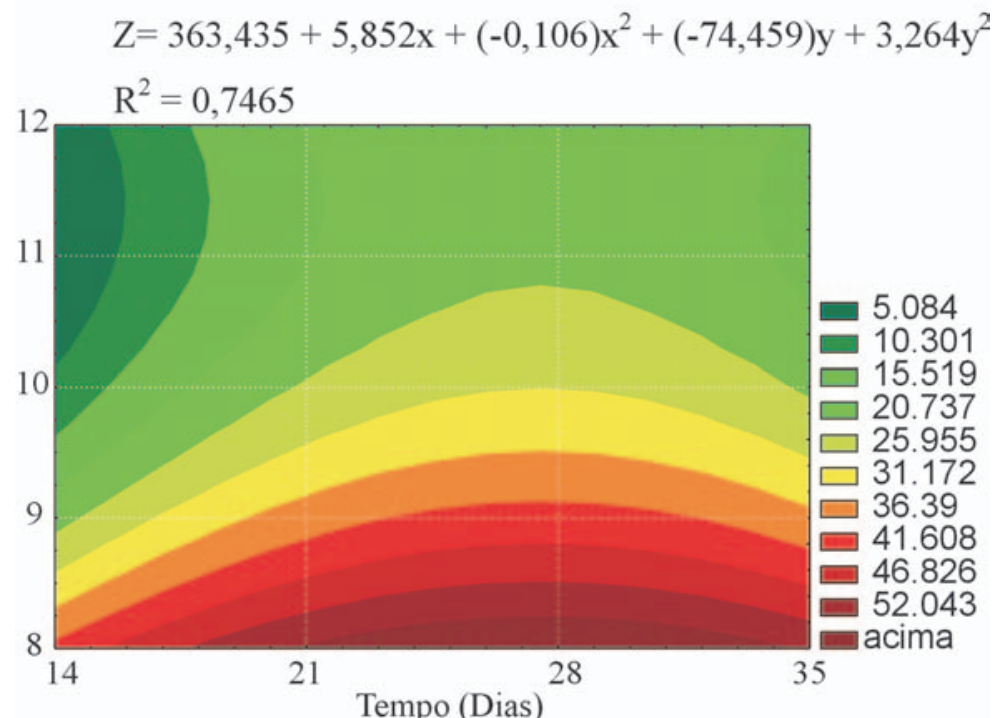

FIGURA 5 - Firmeza de polpa (N) em mamão Formosa 'Tainung 01' mantido a $8 ; 10$ e $12^{\circ} \mathrm{C}$ e $90 \pm 5 \%$ UR durante os períodos de $7 ; 14 ; 21$ e 28 dias, acrescidos de um período de comercialização simulado de 7 dias a $20^{\circ} \mathrm{C}$ e $65 \pm 5 \%$ UR.

\section{CONCLUSÕES}

1. A melhor estimativa para extensão da vida útil pós-colheita do mamão Formosa 'Tainung 01' foi observada nos frutos submetidos a $10^{\circ} \mathrm{C}, 90 \pm 5 \%$ UR até 20 dias, com boa aparência externa e interna, desenvolvimento da coloração amarela na casca, firmeza de polpa superior a $20 \mathrm{~N}$ e perda de massa de $7 \%$.

2. Os frutos submetidos a $8{ }^{\circ} \mathrm{C}$ e a $10{ }^{\circ} \mathrm{C}, 90 \pm 5 \%$ UR desenvolveram sintomas de danos pelo frio após 21 dias de armazenamento, agravando-se com a extensão do armazenamento.

3. No armazenamento a $12{ }^{\circ} \mathrm{C}, 90 \pm 5 \%$ UR, observou-se um amadurecimento acelerado com amolecimento aquoso da polpa a partir de 28 dias. Aos 35 dias, os frutos estavam inadequados para o consumo, 
havendo grande incidência de Colletotrichum gloeosporioides.

\section{REFERÊNCIAS}

ABELES, F.B.; MORGAN, P.W.; SALTVEIT Jr.M. E. Etylene in plantbiology. 2.ed. New York: Academic Press, 1992. 414p.

AN, J.F.; PAULL. R. E. Storage temperature and ethylene influence on ripening of papaya fruit. Journal of the American Society for Horticultural Science, Alexandria, v. 115, n. 6, p. 949-953, 1990.

FAO 2004. Disponível em: <http://www.faostat.org.br > Acesso em: 18 jan. 2004.

HARVEY, T.; CHAN, Jr. Alleviation of chilling injury in papayas. HortScience, Alexandraia, v. 23, n. 5, p. 868-870, 1988.

JANDEL SCIENTIFIC. User's manual. California: Jandel Scientific, 1991. 280p.

LOPES, M.A. Conservação do mamão em condições ambiente submetido a aplicação de cera e cloreto de cálcio. 1997. 39p. Dissertação (Mestrado em Fitotecnia) - Escola Superior de Agricultura de Mossoró, Mossoró, 1997.

MENEZES, J.B.; CASTRO, E.B.; PRAÇA. E.F.; GRANGEIRO, L.C.; COSTA, L.B.A. Efeito do tempo de insolação pós-colheita sobre a qualidade do melão-amarelo. Horticultura Brasileira, Brasília, v.16, n.1, p.80-81, 1998.
RIBEIRO, M.D. Estudos preliminares do comportamento do mamão Formosa armazenado em condições ambientais. 2002. 39p. Monografia (Agronomia) - Escola Superior de Agricultura de Mossoró, Mossoró, 2002.

SAS INSTITUTE. SAS procedures guide for computers. 6.ed., Cary NC, 1993.373p.

SILVA, E. de O. Efeito da embalagem plástica e da temperatura sobre a qualidade pós-colheita do mamão. 1995. 74p. Dissertação (Mestrado em Fitotecnia) - Universidade Federal de Viçosa, Viçosa, 1995.

TEIXEIRA, G.H. deA.; DURIGAN, J.F.; MATTIUZ, B.H.; ROSSI JUNIOR, O.D. Processamento mínimo de mamão Formosa. Ciência \& Tecnologia de Alimentos, Campinas, v. 21, n. 1, p. 47-50, 2001.

WILLS, R. B. H.; WIDJANARKO, S.B. Changes in physiology, composition and sensory characteristics of Australian papaya during ripening. Australian Journal of Experimental Agriculture, Collingwood, n. 35, p. 1173-1176, 1995.

WILLS, R.H.H.; LEE, T.H. ; GRAHAM, D.; McGLASSON, W.B.; HALL, E.G. Postharvest: an introduction to the physiology and handling of fruit and vegetables. London: Granada, 1981. 163p. 